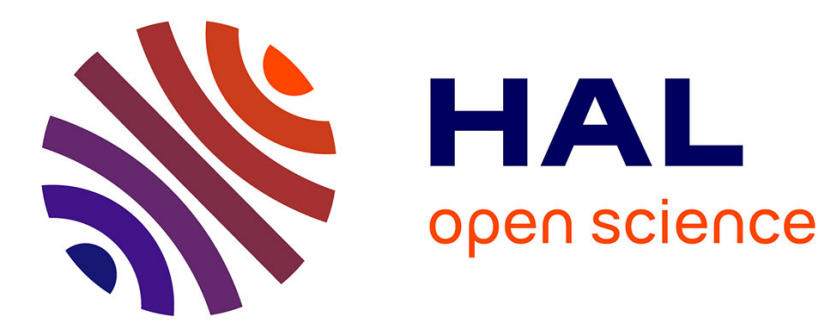

\title{
Réseaux sociaux et coopération entre concurrents
}

Emmanuel Lazega, Elise Penalva-Icher

\section{To cite this version:}

Emmanuel Lazega, Elise Penalva-Icher. Réseaux sociaux et coopération entre concurrents. Hermès, La Revue - Cognition, communication, politique, 2011, 59, pp.43 - 49. 10.3917/herm.059.0043 . hal01800224

\section{HAL Id: hal-01800224 \\ https://hal-sciencespo.archives-ouvertes.fr/hal-01800224}

Submitted on 25 May 2018

HAL is a multi-disciplinary open access archive for the deposit and dissemination of scientific research documents, whether they are published or not. The documents may come from teaching and research institutions in France or abroad, or from public or private research centers.
L'archive ouverte pluridisciplinaire HAL, est destinée au dépôt et à la diffusion de documents scientifiques de niveau recherche, publiés ou non, émanant des établissements d'enseignement et de recherche français ou étrangers, des laboratoires publics ou privés. 
Emmanuel Lazega et Élise Penalva Icher

Université Paris-Dauphine

\section{RÉSEAUX SOCIAUX ET COOPÉRATION ENTRE CONCURRENTS}

«Je ne veux pas faire partie d'un club
qui accepte les gens comme moi comme membre»

Groucho MARX (1959).

\section{Rationalisation de la gestion des interdépendances}

Au début $\mathrm{du} \mathrm{Xx}^{\mathrm{e}}$ siècle, le fait de savoir lire et écrire constituait encore en France un avantage concurrentiel considérable sur les marchés du travail. Un siècle plus tard, dans une société organisationnelle, fortement managériale et marchande, les compétences relationnelles et de gestion des interdépendances relationnelles jouent un rôle similaire. Si ces savoir-faire relationnels et les stratégies individuelles de gestion de ces interdépendances ont acquis cette importance nouvelle, il apparaît aussi que les pratiques liées à ces savoir-faire sont aujourd'hui en voie de recodification et de rationalisation. Plusieurs raisons peuvent expliquer cette évolution. D’une part, les formes de solidarité universalistes qui ont caractérisé les sociétés occidentales après la Seconde Guerre mondiale sont remises en cause. Les risques associés au cycle de vie et à la vie de travail n'étant plus systématiquement pris en charge par le collectif dans son ensemble, la question est reposée de leur partage au niveau méso-social (famille, profession, entreprise, etc.). Ces partages sont beaucoup plus personnalisés et «encastrés » que ceux auxquels la sécurité sociale avait habitué, par exemple, les citoyens français. Les individus sont ainsi contraints de se soucier de leurs échanges sociaux dans un esprit 
nouveau et s'équipent en conséquence de logiciels d'analyse de leur carnet d'adresses, voire de représentation de leurs réseaux personnels socionumériques. La gestion plus ou moins quotidienne ou chronophage des réseaux personnels que sont les profils en ligne disponibles sur les sites les plus répandus actuellement (Facebook, LinkedIn, Viadeo, etc. en France) fait partie de cette tendance. Cette gestion ne relève pas nécessairement d'un «calcul» économique, mais elle fait partie d'une codification nouvelle des échanges sociaux.

D'autre part, la division du travail sophistiquée des économies contemporaines, dans un contexte d'individualisme et de mise en concurrence de plus en plus ouverte des individus, crée des interdépendances et des modes de coordination d'une complexité inédite pour les acteurs au travail. La participation à l'action commune - par exemple, dans le travail en équipe, dans les «partenariats» entre entreprises, dans les «réseaux» professionnels aux contours plus flous - requiert de l'imagination dans les efforts de coordination et dans les échanges de toutes sortes de ressources. À l'échelle de l'individu, ces ressources comprennent les moyens utiles aux fins personnelles, comme par exemple de l'information, de la bonne volonté des collègues, du conseil, ou encore des ressources qui n'ont pas nécessairement de lien direct avec le processus de production: par exemple, le soutien émotionnel ou la définition de la situation. Les relations apparaissent ici comme des canaux pour la circulation de ces ressources hétérogènes (sociales et marchandes) mais aussi comme le lieu d'engagements vis-à-vis de partenaires d'échange. Avec cette dimension symbolique, ces relations acquièrent une certaine régularité et stabilité. Ces engagements peuvent être remis en question mais ils contiennent une promesse, une obligation ou une convention morale introduisant la durée dans les échanges et présupposant un dispositif de contrôle social rendant cette promesse crédible. $\mathrm{Du}$ fait de cette dimension symbolique de la relation sociale, les réseaux sociaux ne peuvent pas être gérés de manière purement utilitariste. Un processus régulatoire est donc en cours de déploiement.

\section{Gestion du capital relationnel de l'individu}

Cette gestion devient particulièrement importante, car c'est elle qui permet à l'acteur de mesurer et de mobiliser son «capital relationnel». C'est dans leurs réseaux de relations que les individus se construisent un répertoire personnel de modes de gestion de ces interdépendances, une sorte de style. Ces styles ne sont pas purement personnels. En effet, le processus régulatoire s'accompagne d'une rationalisation croissante de la gestion des interdépendances. Nous considérons le développement des techniques d'analyse et de visualisation des réseaux sociaux par la sociologie contemporaine ${ }^{1}$, puis la diffusion dans le grand public de ces techniques (dans les logiciels de visualisation des réseaux sociaux en ligne, notamment) comme une évolution relevant dès le départ d'une rationalisation croissante de cette gestion à l'échelle de l'individu. La quête d'efficacité et de contrôle que Max Weber associait à la modernité a fini par gagner un domaine que la société occidentale considère encore comme profondément personnel, intime et privé: la vie relationnelle.

Inévitablement, cette rationalisation s'accompagne d'une redéfinition, voire d'une certaine dilution, des frontières entre la sphère privée et l'espace public. La société organisationnelle exige de plus en plus des individus qu'ils s'appuient sur leurs propres relations informelles et personnalisées, autant que sur les canaux impersonnels et formalisés des couloirs d'action routinisée, pour participer à toutes les formes d'action 
commune ou collective. Cette dynamique de personnalisation/dépersonnalisation des échanges, où l'acteur s'engage de manière plus contingente et variable, s'inscrit de plus en plus systématiquement au cœur de la régulation de l'action. Ce phénomène est aussi l'une des facettes de la décollectivisation contemporaine dans la vie économique et sociale, de la démutualisation de certains risques au sein de la société salariale, des déplacements des frontières entre public/privé ou salariat/ entrepreneuriat (Castel, 1995; Boyer, 2001) mais aussi $\mathrm{du}$ transfert aux individus des coûts d'adaptation aux marchés du travail flexibilisés

Si la rationalisation de la gestion des interdépendances ne peut pas être purement utilitariste, elle relève en partie de ce que nous avons appelé une rationalité sociale (Lazega, 2003). Ainsi, «gérer» des relations (des ressources et des engagements) relève de toute une «discipline sociale» complexe, instrumentale, mais aussi reconnue par les acteurs eux-mêmes comme légitime. Une approche néo-structurale de la rationalité sociale identifie au moins deux dimensions de la rationalité des individus dans la gestion de ces interdépendances: la recherche de niches sociales et l'entrée dans la concurrence de statut social. La gestion des réseaux personnels socionumériques peut fournir des indicateurs précieux sur la mise en œuvre concrète de cette rationalité sociale. Cette rationalité sociale passe ainsi par la construction de niches sociales (définie, en termes d'analyses de réseaux, comme une position dense et multiplexe dans un système de positions) et par l'entrée dans la concurrence de statut social. Dans un univers de «semi-coopérations» sur lequel viennent se greffer les pratiques du monde numérique, la gestion du capital relationnel individuel est elle-même sophistiquée et coûteuse car elle finit, avec le temps, par refléter ou s'adapter à la discipline sociale que l'individu considère comme légitime.

Bien qu'un profil individuel ne soit pas à proprement parler, pour les sociologues, le lieu de reconstitution de systèmes de niches sociales ${ }^{2}$, il visualise pour chaque individu un ensemble de cercles sociaux ou de contextes organisationnels à l'intersection desquels il se positionne de manière visible. Ces cercles peuvent être nombreux ou rares, de grande ou petite taille, denses ou peu denses, etc. Cette gestion du nombre, de la taille ou de la densité est sous-tendue par la dynamique de la construction des réseaux personnels et de la co-évolution, dans ces réseaux, des relations et des comportements de l'acteur, mais aussi des attributs de ses contacts. Bien que les sites jouent beaucoup sur le vocabulaire de la communauté ou de l'amitié propres à la mythologie du partage du Web 2.0, chacun a bien conscience que l'amitié sur Facebook ne correspond pas exactement à une amitié hors connexion: les profils peuvent témoigner d'une plus ou moins grande homogénéité en termes de caractéristiques économiques, macro-sociales, culturelles des contacts. La construction d'un capital relationnel reflète nécessairement certains types d'homophilie et d'hétérophilie, des ressemblances et des différences, dont on peut faire l'hypothèse qu'elle marque fortement cette gestion en ligne, autant en statique qu'en dynamique. C'est par exemple l'hypothèse sur laquelle s'appuie le marketing de la société de consommation contemporaine. Il utilise de manière de plus en plus sophistiquée et ciblée des informations privées sur les relations, les caractéristiques et les historiques d'achats des individus pour obtenir une vue intégrée de leur profil et essayer de diffuser des recommandations personnalisées d'achats ou faire jouer les processus de prescription directe ou indirecte par les pairs (Flichy, 2009).

Comme le montrent les études de la dynamique des réseaux sociaux (Snijders, 2001), les individus peuvent ajouter ou supprimer des relations dans leur profil en ligne pour renforcer des cercles déjà établis en termes de caractéristiques individuelles et sociales (attributs) ou pour diversifier ces cercles. L'évolution des profils s'appuie ainsi sur une co-évolution des relations, des 
attributs et des comportements, co-évolution elle-même dépendante de facteurs endogènes propres à l'échange social (par exemple, la réciprocité ou la transitivité dans les relations) et de facteurs exogènes (par exemple, ces mêmes caractéristiques des individus).

Les réseaux sociaux de l'Internet sont donc utilisés par les individus pour jouer avec leurs cercles sociaux, leurs groupes d'appartenance et de références, pratiquer des comparaisons sociales et culturelles, découvrir et évaluer ainsi le potentiel (de reconnaissance, d'accès, de mobilisation, de coopération, d'échange de ressources) que représente à tout moment leur capital relationnel personnel. Ainsi, le travail que représente la construction et la gestion de ces profils relationnels en ligne correspond de manière encore plus lisible à la seconde dimension de cette rationalité sociale: les jeux de statut lui donnent explicitement une partie de leur sens social, même lorsqu'ils sont ludiques et expérimentaux, comme c'est le cas dans les profils des adolescents et des étudiants - consommateurs importants même s'ils ne sont pas encore entrés pleinement dans les marchés du travail, la vie parentale ou l'activité associative ou politique. Cette gestion correspond donc à une manière d'examiner, d'évaluer, de réévaluer son statut social activée à chaque demande ou acceptation d'une nouvelle relation et au moment de la paramétrisation de la protection de la privacy.

\section{Les jeux de statut social}

Ces jeux de statut social, plus ou moins narcissiques, se lisent en partie dans les pratiques souvent quotidiennes de la mise en valeur de soi en ligne par l'utilisation de dispositifs multiples (profil, blog, job board, galerie de photos, etc.). Comme le note Boyd (2006), l'établissement d'un lien sur le Net dépend du statut de l'autre, du souci de son propre statut et de sa popularité, mais aussi d'obligations sociales liées à la vie hors connexion. On ne peut pas facilement refuser un parent, par exemple, lorsqu'on est un adolescent. Le jeu est d'accroître son statut mais aussi de réfléchir aux risques que l'on encourt en entrant dans les jeux de statut. Pour les générations dites digital natives qui arrivent aujourd'hui sur le marché du travail, les sites de réseaux sociaux d'Internet peuvent servir à gérer systématiquement leur image (self-branding), leur carrière et leurs recrutements. De leur côté, les entreprises font elles-aussi appel à ces réseaux en ligne dans leur gestion du personnel (surveillance fonctionnelle et disciplinaire ${ }^{3}$, imposition d'un mode de vie au travail) ou leur communication. Elles installent leur propre réseau social en interne et les supérieurs hiérarchiques peuvent jeter un œil sur ce qui s'y passe, voire donner des conseils. Ce réseau intraorganisationnel - qui s'ajoute aux réseaux personnels en ligne - impose un mode de vie au travail en particulier avec des collègues à l'étranger et, dans le e-commerce, avec des clients (Allen et al., 2008). Certaines plateformes virtuelles servent à des professionnels de tous types (ingénieurs, informaticiens, commerciaux, scientifiques, entre autres) afin d'établir des communautés de pratiques internationales. Les interactions virtuelles comptent de plus en plus pour les jeux de statut.

Une littérature à la fois sociologique et gestionnaire très abondante se développe sur l'instrumentation et l'«optimisation» des réseaux sociaux individuels en relation avec la concurrence de statut. Le leadership, par exemple, est traduit et mesuré en capacité de créer de la cohésion relationnelle dans les groupes de travail et, simultanément, comme capacité d'intermédiation entre le groupe de travail et son environnement (Burt, 2005). C'est cette concurrence de statut qui peut tomber sous le coup de l'énoncé ironique et en apparence délirant de Groucho Marx (1959): «I don't want to belong to any club that will accept people like me as a member.» 
Cette concurrence est le lieu d'expression des ambitions d'ascension sociale. Pour les individus et familles en mobilité ascendante, les groupes d'appartenance ne correspondent pas aux groupes de référence, les seconds étant plus élevés que les premiers dans la hiérarchie sociale. Dans cette logique utilitariste de club, moins un site est sélectif, et plus les acteurs en cherchent d'autres qui le seront davantage, plus fermés et procurant un environnement supposé plus utile à ces projets de mobilité ascendante. Certains sites cherchent, par exemple, à attirer de nouveaux membres en spécifiant qu'on y liste ses «vrais amis pour la vie» (Friendster) et en jouant sur cette concurrence de statut en proposant des activités hors ligne à de petites audiences sur-sélectionnées et rassemblées en privé (MySpace).

L'analyse des réseaux sociaux, qu'ils soient ou non numérisés, aide les individus et les organisations dans leur recherche d'une gestion rationalisée des interdépendances relationnelles. Mais cette rationalisation accompagne un processus régulatoire très conflictuel: on pense ici aux plaintes déposées devant les tribunaux des prud'hommes pour licenciement abusif après qu'un employé a «publiquement» critiqué son entreprise sur un site de réseau social. De même, on peut s'interroger sur l'absence de droit à l'oubli sur Internet pour des générations d'adolescents qui construisent aujourd'hui une part de leur personnalité sociale via le Web, mais dont l'identité évoluera au cours du cycle de vie. La rationalisation ne peut pas être une simple «ingénierie relationnelle» précisément parce que la gestion des interdépendances fait partie de processus sociaux qui caractérisent les collectifs, qui constituent une discipline sociale complexe leur permettant de se coordonner, constituant aussi une composante du capital social du collectif que l'individu ne maittrise pas seul (Lazega, 2006).

La revendication de ce statut social repose en partie sur l'identification à des autruis et à des cercles ou organisations auxquels on appartient ou auxquels on se réfère. Pour une approche néo-structurale, ces jeux de statut dans la gestion des interdépendances devraient donc se refléter dans les analyses des structures relationnelles. Ces dernières résultent, entre autres, du travail des acteurs sur leur profil relationnel numérique, des disciplines sociales qu'ils considèrent comme légitimes et des styles de gestion des profils qui en résultent. La revendication de statut peut avoir plusieurs dimensions qui permettent d'aboutir à une classification des manières «rationalisées » de gérer ses interdépendances.

\section{Capital relationnel de l'individu et capital social des collectifs}

L'analyse de réseaux met en évidence le travail relationnel et symbolique des acteurs préalablement désolidarisés, une rationalisation sociale qui les guide, les rouages relationnels d'une discipline sociale complexe qui leur permet de se coordonner et qui varie selon les collectifs auxquels ils appartiennent. Il ne s'agit pas de présupposer un «calcul» purement individuel des acteurs dans leur manière de gérer leurs relations et leurs interdépendances. Si les individus sont bien contraints à une rationalisation de leurs échanges sociaux, cette rationalisation ne relève pas nécessairement d'un calcul économique, car l'échange social n'est pas un échange marchand. Cette gestion relève précisément de ce que nous avons appelé une discipline sociale qui force l'individu à une certaine distanciation, à une réflexion sur sa relation aux cercles et organisations dont il est ou a été membre, mais qui facilite aussi le déploiement des processus sociaux sans lesquels il n'y a pas d'action collective. 
Cette notion de discipline sociale fait le lien entre capital relationnel de l'individu - celui qu'il peut tenter de gérer sur Internet - et ce capital social de chacun des collectifs dont il fait partie. La présence en ligne reflète en partie la manière dont les individus, dans leur contexte social, conçoivent cette discipline sociale. Les réseaux sociaux numériques sont donc des indicateurs partiels des formes que prennent les processus fondamentaux de la vie sociale: par exemple la solidarité, le contrôle, la régulation, l'apprentissage et la socialisa-

\section{NOTES}

1. L'analyse des réseaux sociaux en tant que méthode sociologique d'études des processus sociaux (Lazega, 1998 [2007]) tend à devenir une technique et une approche ordinaire en sciences sociales. Son application aux profils des réseaux personnels se généralise aussi bien dans des applications spécialisées offertes aux utilisateurs que dans la recherche sociologique. Voir par exemple Boyd et Ellison, 2007; Casilli, 2010; Georges, 2009; Hogan, 2008; Kumar et al., 2006; Lewis et al., 2008; Roth et al., 2008; Steinfeld et al., 2008; Subramanyam et al., 2008.

\section{RÉFÉRENCES BIBLIOGRAPHIQUES}

Allen, S., Deragon, J. T., Orem, M. G. et Smith, C. F., The Emergence of The Relationship Economy: The New Order of Things to Come, Silicon Valley, Link To Your World Publications, 2008.

Boyd, D., «Friends, Friendster and Top 8: Writing Community into Being on Social Network Sites », First Monday, vol. 12, n 11, 2006. En ligne sur <http://firstmonday.org>, consulté le 02/02/2011.

Boyd, D. et Ellison, N., «Social Network Sites: Definition, History and Scholarship », Journal of computer-mediated communication, vol. 1, n ${ }^{\circ} 13,2007$. En ligne sur $<$ http://jcmc.indiana.edu>, consulté le 02/02/2011. tion, etc. Les avancées de la recherche sur les réseaux sociaux et organisationnels peuvent être mises à profit pour identifier ces formes de discipline sociale qui émergent de ces nouvelles pratiques de construction d'une présence en ligne. Cette question est celle de la contribution de la gestion des profils au fonctionnement de ces processus sociaux considérés comme un atout collectif, une composante du capital social du collectif (et non plus seulement du capital relationnel de l'individu). Dans ce domaine, tout ou presque reste à faire.

2. Une niche sociale est définie, en termes d'analyses de réseaux, comme une position dense et multiplexe dans un système de positions. Techniquement, cette reconstitution n'a de sens qu'à l'échelle du collectif où le système de niches sociales reflète une division du travail dans le système d'échange de ressources sociales.

3. Un débat a lieu actuellement en Allemagne pour interdire cette surveillance des entreprises sur leurs nouvelles recrues et salariés.

BOYER, R., Du rapport salarial fordiste à la diversité des relations salariales, Paris, CEPREMAP, 2001.

BurT, R. S., Brokerage and Closure: An Introduction to Social Capital, New York, Oxford University Press, 2005.

CAsilli, A., Les Liaisons numériques, vers une nouvelle sociabilité?, Paris, Seuil, coll. «La couleur des idées», 2010.

CASTEL, R., Les Métamorphoses de la question sociale, Paris, Fayard, 1995. 
Flichy, P., «Comment Internet est devenu un marché» in Steiner, P. et VAtin F. (dir.), Traité de sociologie économique, Paris, PUF, coll. «Quadrige, 2 2009, p. 451-491.

Georges, F., «Représentation de soi et identité numérique», Réseaux, vol. 2, n 154, 2009, p. 165-193.

Hogan, B., «Analysing Social Networks via the Internet» in Fielding, N., Ray L. et Grant, B., The Handbook of Online Research Methods, Thousand Oaks, Sage, 2008.

Kumar, R., Novak, J. et Tomkins, A., «Structure and Evolution of Online Networks », Proceedings of $12^{\text {th }}$ International Conference on Knowledge Discovery and Data Mining, New York, ACM Press, p. 611-617.

LAZEGA, E., «Rationalité, discipline sociale et structure», Revue française de sociologie, 2003, n 44, p. 305-330.

LAZEGA, E., Réseaux sociaux et structures relationnelles, Paris, PUF, coll. «Que sais-je?», 2007 (1e éd. 1998).

LAZEGA, E., «Réseaux sociaux» in Bevort, A., Jobert, A. et al. (dir.), Dictionnaire du travail, Paris, PUF, 2011.

LAZEGA, E., «Capital social, processus sociaux et capacité d'action collective» in Bevort, A. et Lallement, M. (dir.), Capital social: Échanges, réciprocité, équité, Paris, La Découverte, 2006, p. 213-225.
Lewis, K., Kaufman, J., Gonzalez, M., Wimmer, A. et ChrisTAKIS, N., «Tastes, Ties, and Time: A New Social Network Dataset Using Facebook.com», Social Networks, vol. 30, 2008, p. 330-342.

Lin, N., Social Capital: A Theory of Social Structure and Action, Cambridge, Cambridge University Press, 2001.

Lin, N. et Dumin, M., «Access to Occupations through Social Ties », Social Networks, $n^{\circ} 8,1986$, p. 365-385.

MarX, G., Groucho and Me, New York, Virgin Books, 1959.

Roth, C., Taraborelli, D. et Gilbert, N., «Démographie des communautés en ligne», Réseaux, vol. 6, n 152, 2008, p. 205-240.

SNijDERS, T.A.B, «The Statistical Evaluation of Social Network Dynamics» in Sobel, M. E. et BecKer, M. P., Sociological Methodology, Boston, Londres, Basil Blackwell, 2001, p. 361-395.

Steinfield, C., Ellison, N. et Lampe, C., «Social Capital, SelfEsteem, and Use of Online Social Network Sites: A Longitudinal Analysis», Journal of Applied Developmental Psychology, $\mathrm{n}^{\circ} 29$, 2008, p. 434-445.

Subrahmanyam, K., Reich, S. M., Waechter, N. et Espinoza, G., «Online and Offline Social Networks: Use of Social Networking Sites by Emerging Adults», Journal of Applied Developmental Psychology, nº 29, 2008, p. 420-433. 